\title{
Transport of energetic electrons in a magnetically expanding helicon double layer plasma
}

\author{
Kazunori Takahashi, ${ }^{1,2,3, a)}$ Christine Charles, ${ }^{1}$ Rod Boswell, ${ }^{1}$ Wes Cox, ${ }^{1}$ and
Rikizo Hatakeyama \\ ${ }^{1}$ Space Plasma, Power and Propulsion Group, Research School of Physics and Engineering, The Australian \\ National University, Canberra 0200, Australia \\ ${ }^{2}$ Department of Electronic Engineering, Tohoku University, Sendai 980-8579, Japan \\ ${ }^{3}$ Department of Electrical and Electronic Engineering, Iwate University, Morioka 020-8551, Japan
}

(Received 15 February 2009; accepted 24 April 2009; published online 13 May 2009)

\begin{abstract}
Peripheral magnetic field lines extending from the plasma source into the diffusion chamber are found to separate two regions of Maxwellian electron energy probability functions: the central, ion-beam containing region with an electron temperature of $5 \mathrm{eV}$, and region near the chamber walls with electrons at $3 \mathrm{eV}$. Along the peripheral field lines a bi-Maxwellian population with a hot tail at $9 \mathrm{eV}$ is shown to both originate from electrons in the source traveling downstream across the double layer and correspond to a local maximum in ion and electron densities. (C) 2009 American Institute of Physics. [DOI: 10.1063/1.3136721]
\end{abstract}

The formation of current-free electric double layers ${ }^{1}$ (DLs) and subsequent acceleration of ions ${ }^{2,3}$ in magnetically expanding, rf plasmas have been the subject of vigorous research in the electric propulsion community, due in part to the potential for constructing an innovative, long-lived plasma thruster: the helicon double layer thruster ${ }^{4}$ (HDLT). Investigation into the formation of DLs and the related ionic and electronic behavior in one dimension have been performed experimentally, ${ }^{1-10}$ analytically, ${ }^{11-13}$ and through simulation. ${ }^{5,14,15}$ Further development of the HDLT now requires investigation into the two-dimensional nature of the related physics, including the effects of magnetic fields and mechanical geometry. ${ }^{8}$

Experiments have previously characterized the beam and bulk ions two dimensionally using retarding field energy analyzers (RFEAs) and Langmuir probes. ${ }^{3,16-20}$ Ions accelerated by DLs in low-pressure expanding plasmas are weakly divergent and magnetically detached about $10 \mathrm{~cm}$ downstream of the double layer. ${ }^{16-18}$ The physical mechanisms leading to the dense ring of bulk plasma observed in Langmuir probe measurements downstream of the double layer has been unclear. Measurements of the electron energy probability functions (EEPFs) on the center axis have been carried out to examine the influence of the DL on electrons. ${ }^{10}$ The resulting upstream EEPF reveals a depleted tail corresponding to the downstream EEPF indicating energetic electrons upstream of the DL possess sufficient energy to overcome the potential drop of the DL and neutralize the beam of ions accelerated by the DL, in good agreement with one-dimensional particlein-cell simulations (with three-dimensional treatment of collisions). ${ }^{15}$ Subsequent radial measurements of the EEPF upstream of the DL have demonstrated a significant distinction between the central and peripheral EEPFs. ${ }^{21}$

In the present study, a region of bi-Maxwellian EEPF, accompanying an increase in the measured ion and electron density, is identified which separates two regions of Maxwellian EEPFs: a cold electron population near the chamber

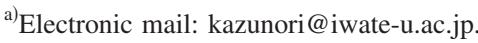

walls and a hotter Maxwellian population in the central region where the ion beam is detected.

The Chi-Kung experimental setup has been described previously ${ }^{21}$ and is shown in Fig. 1(a). It consists of a 31$\mathrm{cm}$-long, 13.7-cm-diameter helicon source attached contiguously to a grounded $29.4-\mathrm{cm}$-long, 31.8-cm-diameter diffusion chamber at a base pressure of a few $10^{-6}$ torr. The argon gas is introduced via a chamber side port and held at an operating pressure of 0.3 mTorr, measured using a Baratron gauge. The source consists of a Pyrex tube surrounded by a double-saddle antenna supplied with $250 \mathrm{~W}$ radio frequency (rf) power at $13.56 \mathrm{MHz}$. Surrounding the source

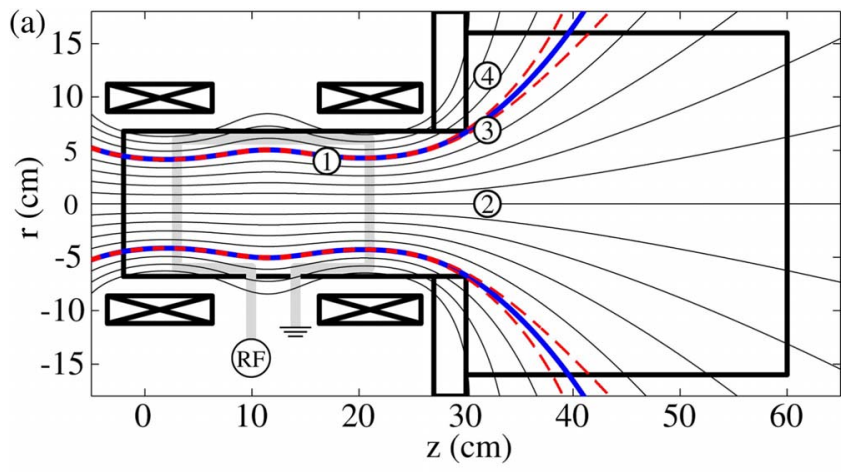

(b)

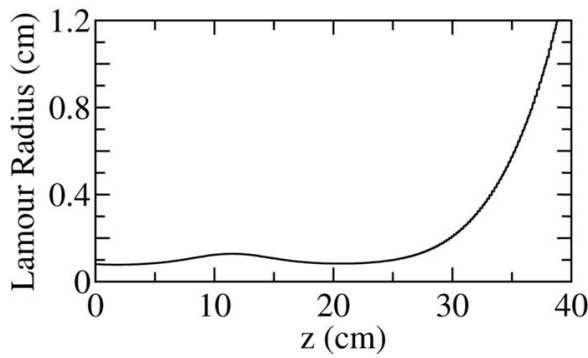

FIG. 1. (Color online) (a) Schematic of Chi-Kung helicon double layer reactor showing the magnetic-field lines (solid black lines), the last field line (bold blue lines), and the calculated Larmour radius of $9 \mathrm{eV}$ electrons along the last field line (dashed red lines). Circles 1-4 correspond to the CP positions of Figs. 2(a)-2(d), respectively. (b) The calculated Larmour radius of $9 \mathrm{eV}$ electrons along the last field line. 

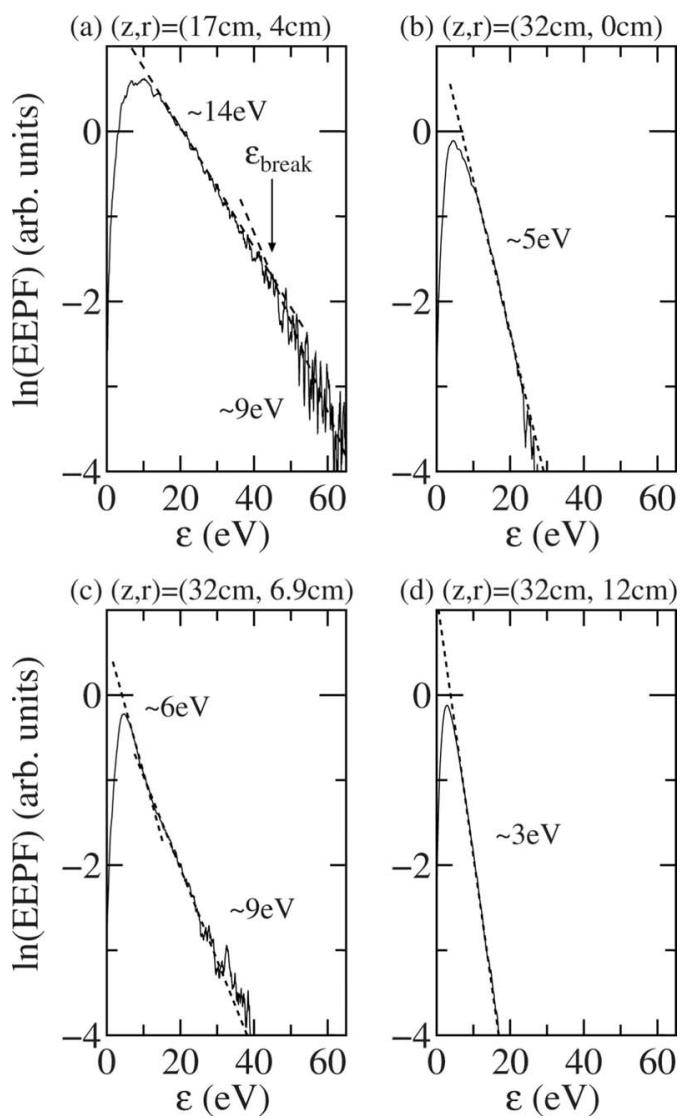

FIG. 2. EEPFs measured using the $\mathrm{CP}$ positioned at (a) $(z, r)$ $=(17 \mathrm{~cm}, 4 \mathrm{~cm})$ [circle 1 in Fig. 1(a)], (b) $(z, r)=(32 \mathrm{~cm}, 0 \mathrm{~cm})$ [circle 2 in Fig. 1(a)], (c) $(z, r)=(32 \mathrm{~cm}, 6.9 \mathrm{~cm})$ [circle 3 in Fig. 1(a)], and (d) $(z, r)=(32 \mathrm{~cm}, 12 \mathrm{~cm})$ [circle 4 in Fig. 1(a)].

tube and antenna, two solenoids placed at $z=3 \mathrm{~cm}$ and 21 $\mathrm{cm}$ produce a magnetic field diverging from a maximum of about $130 \mathrm{G}$ in the source to a few tens of gauss in the diffusion chamber, shown as solid lines in Fig. 1(a). Under these operating conditions, a DL spontaneously forms near the open end of the source tube at $z=25 \mathrm{~cm}$ (Ref. 1) with an amplitude of $25 \mathrm{~V}$. Consequently, an RFEA or laser-induced fluorescence can be used to detect the presence of an accelerated beam of ions in the diffusion chamber. ${ }^{3,5}$

Measurements of the EEPF are performed using a 3-mm-long, 0.25-mm-diameter cylindrical rf-compensated Langmuir probe $(\mathrm{CP})$ attached at an angle to a rotatable rod, previously described, ${ }^{10,21}$ permitting measurements in a circular arc. A simple trigonometric calculation converts this arc to a radial measurement. Sweeping the probe bias voltage allows the ion current to be measured as a function of voltage, the $I-V$ characteristic. Using an active analog circuit, the $I-V$ characteristic can be twice differentiated to produce the EEPF. Spatial measurements of the ion energy distribution function and ion density are performed in the diffusion chamber with a similarly rotating RFEA. ${ }^{18}$

Figure 2(a) shows the EEPF at $(z, r)=(17 \mathrm{~cm}, 4 \mathrm{~cm})$, the region where magnetic field lines terminate in the source tube walls and do not extend into the diffusion chamber. Previous measurements in this region have reportedly exhibited depletion from a Maxwellian distribution at a break energy of $\varepsilon_{\text {break }}=45 \mathrm{eV},{ }^{21}$ indicated as an arrow in Fig. 2(a). As a consequence of the rf heating effect in the helicon source, above and below the break energy the average elec- tron energy is $9 \pm 1.5$ and $14 \pm 1 \mathrm{eV}$, respectively. The break energy is determined as the intersection point of two tangential lines providing these average energies. The detailed radial measurement of the EEPF previously performed at the upstream source plasma ${ }^{21}$ has shown that electrons are trapped by the magnetic field lines terminating in the source wall. As a consequence, the resulting break energy is in good agreement with the sheath amplitude at the Pyrex glass wall of about $46 \mathrm{~V}^{21}$ while the central EEPF has shown a depletion of about $27 \mathrm{eV}$ in the source, corresponding to the potential drop of the DL. ${ }^{10}$

Radial measurements of the EEPF in the region downstream of the DL (Fig. 2), reveal the presence of three distinct EEPFs: at the center $(z, r)=(32 \mathrm{~cm}, 0 \mathrm{~cm})$, near the outermost magnetic field line not intersecting the source wall [the "last field line," blue bold line in Fig. 1(a)] $(z, r)$ $=(32 \mathrm{~cm}, 6.9 \mathrm{~cm})$, and beyond the last field line $(z, r)$ $=(32 \mathrm{~cm}, 12 \mathrm{~cm})$. In agreement with previous work, ${ }^{10}$ the EEPF in Fig. 2(b) shows a Maxwellian distribution at the radial center with a temperature of about $5 \mathrm{eV}$, analogous to the depleted tail of the upstream EEPF. The bi-Maxwellian distribution near the last field line shown in Fig. 2(c) indicates a population of energetic electrons with a temperature of about $9 \mathrm{eV}$, similarly analogous to the depleted tail of the upstream EEPF in Fig. 2(a) measured inside the terminating field lines. It is considered that this population of $9 \mathrm{eV}$ electrons in Fig. 2(c) originated from the upstream population of depleted tail electrons with sufficient energy to overcome the potential of the DL which then travel along the last field line into the diffusion chamber. In the region beyond the last field line, where the magnetic field lines terminate in the diffusion chamber walls and do not enter the source region, a Maxwellian population of $3 \mathrm{eV}$ cold electrons are observed [Fig. 2(d)], likely created in the downstream ionization region surrounding the last field line and then collisionally dissipating into this magnetically isolated region.

Detailed radial CP and RFEA measurements are performed at $z=32 \mathrm{~cm}$ to probe the electron and ion populations downstream of the double layer and investigate the local maximum in the total ion density previously measured at about $r=8 \mathrm{~cm}$ with a Langmuir probe. ${ }^{3,19}$ Transport of electrons, with energy higher than the ionization energy (about $16 \mathrm{eV}$ ), from the source along the last magnetic field line may explain the local ion density maximum reported in Refs. 3 and 19, through an increase in the ionization downstream of the DL. The observed Maxwellian or biMaxwellian EEPFs can be characterized by one (bulk) or two (bulk and tail) electron temperatures, $T_{e}$ bulk and $T_{e}$ tail, shown in Fig. 3(a). The electron density $n_{e}$ (integral of the EEPF) and measured beam, $I_{\text {beam }}$, and total (discriminator voltage $=0 \mathrm{~V}), I_{\text {total }}$, current from the RFEA are plotted in Fig. 3(b) as open squares, crosses, and triangles, respectively. Within the diffusion chamber core $(r<6 \mathrm{~cm})$ the EEPFs exhibit a Maxwellian distribution of about $6 \mathrm{eV}$, mirroring the upstream EEPF above the break energy corresponding to the potential drop of the DL. The bi-Maxwellian distribution characterized by $T_{e}$ bulk and $T_{e}$ tail is observed in the region surrounding the last magnetic field line $(6 \mathrm{~cm}<r<10 \mathrm{~cm})$, while beyond this region $(r>10 \mathrm{~cm})$ the bulk temperature $T_{e}$ bulk is observed to gradually decrease. In order to discuss the width of the area where the hot tail electrons are detected, the Lamour radius of the $9 \mathrm{eV}$ electrons are calculated along 

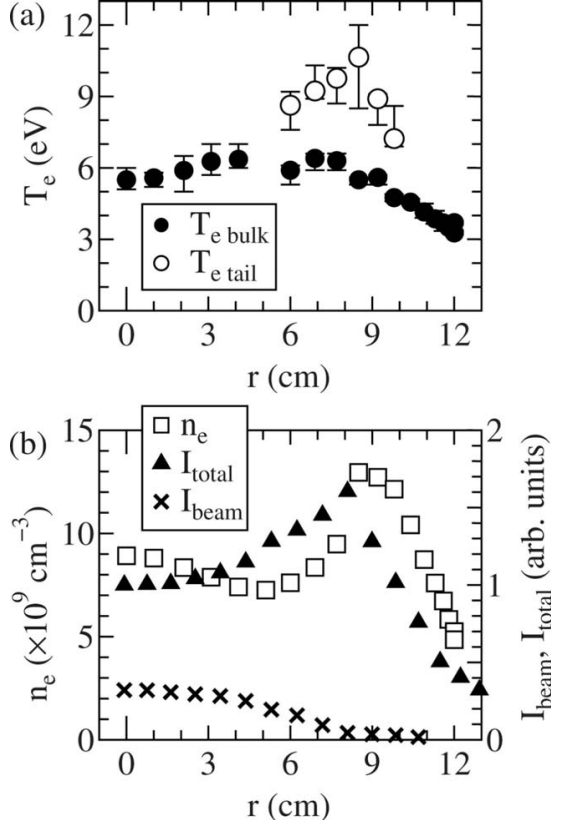

FIG. 3. Radial profiles of (a) electron temperatures $T_{e}$ bulk (closed circles) and $T_{e}$ tail (open circles), and (b) electron density $n_{e}$ (open squares) measured with the rf compensated Langmuir probe, ion beam current $I_{\text {beam }}$ (crosses) and ion total current $I_{\text {total }}$ (triangles) measured with the RFEA.

the last field line [Fig. 1(b)], and the electron orbit radius along the field line is plotted as dashed red lines in Fig. 1(a). The length of the CP tip is $3 \mathrm{~mm}$ and the Lamour radius is about $3 \mathrm{~mm}$ at $(z, r)=(32 \mathrm{~cm}, 8 \mathrm{~cm})$. Under the present operating conditions $\left(n_{e}=10^{10} \mathrm{~cm}^{-3}\right.$ and $\left.T_{e}=9 \mathrm{eV}\right)$ the Debye length $\lambda_{\mathrm{De}}$ is approximately $0.25 \mathrm{~mm}$. Because the sheath voltage is large compared to $T_{e}$, the sheath length is known to be tens of Debye lengths such that the sheath around the probe tip is approximately $1 \mathrm{~cm}$ in extent. ${ }^{22}$ Thus the summation of the Larmour radius, probe tip and the sheath length are approximately equal to the width of the energetic tail electrons shown in Fig. 3(a), indicating that the local ion density peak in the diffusion chamber is likely due to some transport of energetic electrons from the source along the last field line into the diffusion chamber.

The beam current $I_{\text {beam }}$ in Fig. 3(b) shows the parabolic profile as previously reported; ${ }^{3,18}$ while both the total current $I_{\text {total }}$ and electron density $n_{e}$ are found to have peaks at around $r=8 \mathrm{~cm}$, confirming the presence of the local maximum of bulk ions and electrons. ${ }^{19}$ Although the particle transport across the magnetic field lines is complex, it is likely that the local maximum in the measured electron den- sity (with the $\mathrm{CP}$ ) and ion density (with the RFEA) would result from a local increase of ionization from the $9 \mathrm{eV}$ population. This is consistent with the observed cold population of $3 \mathrm{eV}$ electrons which lack the required energy to overcome the DL and would otherwise remain trapped upstream in the source.

In summary, three populations of electrons are measured downstream of the DL. The first are electrons originating from the depleted tail of the central upstream population, traveling downstream to neutralize the ion beam. The second population derive from the energetic population of peripheral electrons transported from the source downstream across the DL along the last field line increasing the ionization (and local ion and electron densities) in the region $r \sim 8 \mathrm{~cm}$ at $z$ $\sim 32 \mathrm{~cm}$. Radially beyond these is the third population of outlying cold electrons collisionally cooled from the energetic electronic population.

${ }^{1}$ C. Charles and R. Boswell, Appl. Phys. Lett. 82, 1356 (2003).

${ }^{2}$ S. A. Cohen, N. S. Siefert, S. Stange, R. F. Boivin, E. E. Scime, and F. M. Levinton, Phys. Plasmas 10, 2593 (2003).

${ }^{3}$ C. Charles and R. W. Boswell, Phys. Plasmas 11, 1706 (2004).

${ }^{4}$ M. D. West, C. Charles, R. W. Boswell, and J. Propul, Power 24, 134 (2008)

${ }^{5}$ X. Sun, A. M. Keesee, C. Biloiu, E. E. Scime, A. Meige, C. Charles, and R. W. Boswell, Phys. Rev. Lett. 95, 025004 (2005).

${ }^{6}$ N. Plihon, P. Chabert, and C. S. Corr, Phys. Plasmas 14, 013506 (2007).

${ }^{7}$ C. Charles, Plasma Sources Sci. Technol. 16, R1 (2007).

${ }^{8}$ I. A. Biloiu, E. E. Scime, and C. Biloiu, Appl. Phys. Lett. 92, 191502 (2008).

${ }^{9}$ K. Takahashi, K. Oguni, H. Yamada, and T. Fujiwara, Phys. Plasmas 15, 084501 (2008).

${ }^{10}$ K. Takahashi, C. Charles, R. W. Boswell, T. Kaneko, and R. Hatakeyama, Phys. Plasmas 14, 114503 (2007).

${ }^{11}$ F. F. Chen, Phys. Plasmas 13, 034502 (2006).

${ }^{12}$ M. A. Lieberman and C. Charles, Phys. Rev. Lett. 97, 045003 (2006).

${ }^{13}$ M. A. Lieberman, C. Charles, and R. W. Boswell, J. Phys. D 39, 3294 (2006).

${ }^{14}$ A. Meige, R. W. Boswell, C. Charles, and M. M. Turner, Phys. Plasmas 12, 052317 (2005).

${ }^{15}$ A. Meige and R. W. Boswell, Phys. Plasmas 13, 092104 (2006).

${ }^{16}$ C. Charles, IEEE Trans. Plasma Sci. 33, 336 (2005).

${ }^{17}$ C. Charles, R. W. Boswell, and M. A. Lieberman, Appl. Phys. Lett. 89, 261503 (2006).

${ }^{18}$ W. Cox, C. Charles, R. W. Boswell, and R. Hawkins, Appl. Phys. Lett. 93, 071505 (2008).

${ }^{19}$ W. Cox, R. Hawkins, C. Charles, and R. W. Boswell, IEEE Trans. Plasma Sci. 36, 1386 (2008).

${ }^{20}$ K. Takahashi and T. Fujiwara, Appl. Phys. Lett. 94, 061502 (2009).

${ }^{21}$ K. Takahashi, C. Charles, R. W. Boswell, and R. Hatakeyama, Phys. Plasmas 15, 074505 (2008).

${ }^{22}$ M. A. Lieberman and A. J. Lichtenberg, Principles of Plasma Discharges and Material Processing, 2nd ed. (Wiley, New York, 2005), Chap. 6. 